\title{
Near IR imaging of Centaurus group dwarf elliptical galaxies
}

\author{
M. Rejkuba ${ }^{1}$, H. Jerjen ${ }^{2}$, G. Da Costa ${ }^{2}$, B. Binggeli ${ }^{3}$ \\ and M. Zoccali ${ }^{4}$ \\ ${ }^{1}$ European Southern Observatory, Karl-Schwarzschild-Strasse 2, D-85748 Garching, Germany \\ email: mrejkuba@eso.org \\ ${ }^{2}$ Research School of Astronomy and Astrophysics, Australian National University, Mount \\ Stromlo Observatory, Cotter Road, Weston ACT 2611, Australia \\ ${ }^{3}$ Astronomical Institute of the Univ. of Basel, Venusstrasse 7, CH-4102 Binningen, Switzerland \\ ${ }^{4}$ P. Univ. Católica de Chile, Astronomy \& Astrophysics Dept., Casilla 306, Santiago 22, Chile
}

\begin{abstract}
Detailed studies of dwarf galaxies in the Local Group have revealed an amazing diversity in their star formation histories. Young and intermediate-age stellar populations are observed in some gas-poor dwarf ellipticals, like Fornax and Carina, where naively one would have expected to find preferentially old stellar populations. There are evidences that the environment might drive the evolution of these galaxies.

In order to explore stellar populations in dwarf galaxies in an environment denser than that of the Local Group we have observed 14 dwarf elliptical galaxies in Centaurus group in nearIR $J_{s}$ and $K_{s}$ bands. The intrinsic properties of these galaxies, the metallicities and ages of their stellar populations and the presence or absence of bright, intermediate-age AGB stars will be correlated with extrinsic properties like the local galaxy density. Here we summarize our observations, present the sample and show near-IR color-magnitude diagrams and luminosity functions for ESO269-066 and AM1343-452 dwarf galaxies.
\end{abstract}

Keywords. galaxies: dwarf, galaxies: fundamental parameters, galaxies: photometry, galaxies: stellar content

\section{The Data}

Deep $J_{s}$ and $K_{s}$ band images for 14 Centaurus Group dwarf galaxies were obtained with the short wavelength arm of ISAAC near-IR array at Antu (UT1) Very Large Telescope (VLT) at ESO Paranal Observatory. Field of view of the short wavelength arm of ISAAC is $2.5 \times 2$ '.5 and the detector is Hawaii Rockwell array with a pixel scale of $0 . \prime 148$. The list of all the targets is given in the Table 1 . The observations were carried out in service mode between April and September 2004. Each galaxy was observed once in $J_{s}$ with a total exposure of $35 \mathrm{~min}$, and twice in $K_{s}$ band with a total exposure of $72 \mathrm{~min}$. Seeing was typically between $0 . .4-0 . \prime 6$ and only for some images up to $0 . \prime 8$. The good seeing and high sensitivity of ISAAC allowed us to resolve well upper red giant branch stars in 7 galaxies, while for the other targets we could resolve only few brightest stars.

The images were reduced using IRAF. For all the targets PSF fitting photometry was done using the suite of DAOPHOT and ALLFRAME programmes (Stetson 1994). The photometry was then tied to 2MASS photometric system by matching all the point sources from 2MASS observed in our fields with our $J_{s}$ and $K_{s}$ band detections. 
Table 1. List of the 14 dwarf galaxies observed with ISAAC. Columns 4 and 5 report distance moduli (DM) from the literature, and the methods used to derive them: TRGB=Tip of the red giant branch, $\mathrm{SBF}=$ surface brightness fluctuation, $\mathrm{MEM}=$ average distance to the group whose membership is based on the morphology. The last column lists references to the literature where these distances were originally published (see the list of references for details).

\begin{tabular}{lccrcll}
\hline \multicolumn{1}{c}{ Galaxy } & RA $_{J 2000}$ & DEC $_{2000}$ & \multicolumn{1}{c}{ Type } & DM & method & Reference \\
\hline AM1320-230 & $13: 23: 29.8$ & $-23: 23: 35.0$ & $\mathrm{dE}$ & 28 & MEM & JBF00 \\
AM1339-445 & $13: 42: 05.8$ & $-45: 12: 20.9$ & $\mathrm{dE}$ & $27.77,27.87$ & TRGB, SBF & K+02, JFB00 \\
AM1343-452 & $13: 46: 17.8$ & $-45: 41: 04.9$ & $\mathrm{dE}$ & $27.92,27.99$ & TRGB, SBF & K+02, JFB00 \\
Cen8 & $13: 22: 56.2$ & $-33: 34: 19.9$ & $\mathrm{Im} / \mathrm{dE}$ & 28 & MEM & JBF00 \\
CenA-dE1 & $13: 12: 47.7$ & $-41: 50: 03.0$ & $\mathrm{dE}$ & 28 & MEM & JBF00 \\
CenA-dE2 & $13: 21: 31.9$ & $-31: 53: 12.0$ & $\mathrm{dE} / \mathrm{Im}$ & 28 & MEM & JBF00 \\
CenA-dE3 & $13: 46: 00.7$ & $-36: 19: 45.0$ & $\mathrm{dE}$ & 28 & MEM & JBF00 \\
CenA-dE4 & $13: 46: 39.6$ & $-29: 58: 42.9$ & $\mathrm{dE}$ & 28 & MEM & JBF00 \\
CenA-dE5 & $14: 30: 04.9$ & $-33: 28: 45.0$ & $\mathrm{dE}$ & 28 & MEM & JBF00 \\
ESO219-010 & $12: 56: 09.6$ & $-50: 08: 38.0$ & $\mathrm{dE}, \mathrm{N}$ & 28.34 & $\mathrm{SBF}$ & JFB00 \\
ESO269-066 & $13: 13: 07.9$ & $-44: 53: 21.9$ & $\mathrm{dE}, \mathrm{N}:$ & 27.75 & SBF & JFB00 \\
ESO384-016 & $13: 57: 01.2$ & $-35: 19: 59.0$ & $\mathrm{dS0} / \mathrm{Im}$ & 27.85 & $\mathrm{SBF}$ & JFB00 \\
SGC1319.1-4216 & $13: 22: 00.4$ & $-42: 32: 08.0$ & $\mathrm{dE}$ & 28 & MEM & JBF00 \\
UGCA365 & $13: 36: 30.7$ & $-29: 14: 11.0$ & $\mathrm{dIrr} / \mathrm{dE}$ & 28 & MEM & JBF00 \\
\hline
\end{tabular}
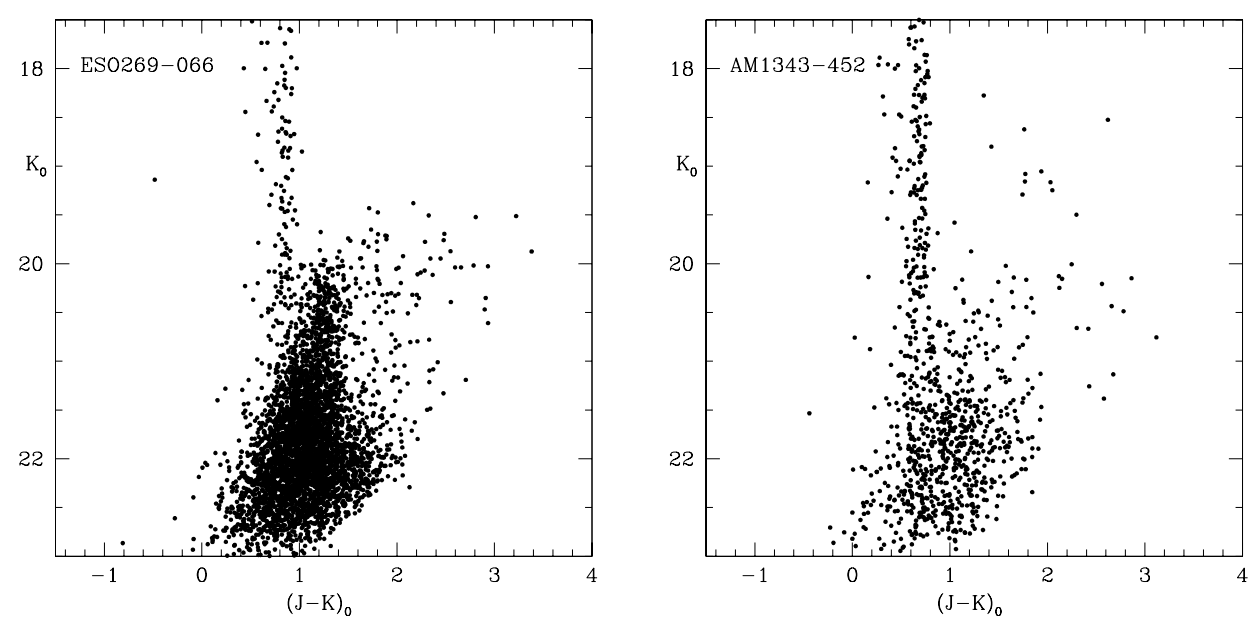

Figure 1. Color-magnitude diagrams of ESO269-066 and AM1343-452.

\section{Near-IR color-magnitude diagrams and luminosity functions}

Matching the $J_{s}$ and $K_{s}$ band photometry color-magnitude diagrams (CMDs) were produced for all the stars in each galaxy field. Near-IR color-magnitude diagrams of two well resolved dwarf galaxies ESO269-066 and AM1343-452 are presented in Fig. 1, showing well populated upper RGB, and possibly some evidence of intermediate-age AGB stars. Due to rather low galactic latitude of our targets $\left(b \sim 20^{\circ}\right)$ there is significant galactic foreground contamination. Most of the Galactic stars have colors between $(J-$ $K)_{0}=0.65-0.85$ and can be easily seen as the vertical sequence at these colors.

Assuming the distance modulus from the literature (see Tab. 1), we can determine the average metallicity of the RGB stars from the mean $(J-K)_{0}$ color at $M_{K}=-5.5$ (Valenti et al. 2004). It is $[\mathrm{Fe} / \mathrm{H}]=-0.65 \pm 0.15$ for ESO269-066, and $-1.1 \pm 0.3 \mathrm{dex}$ 

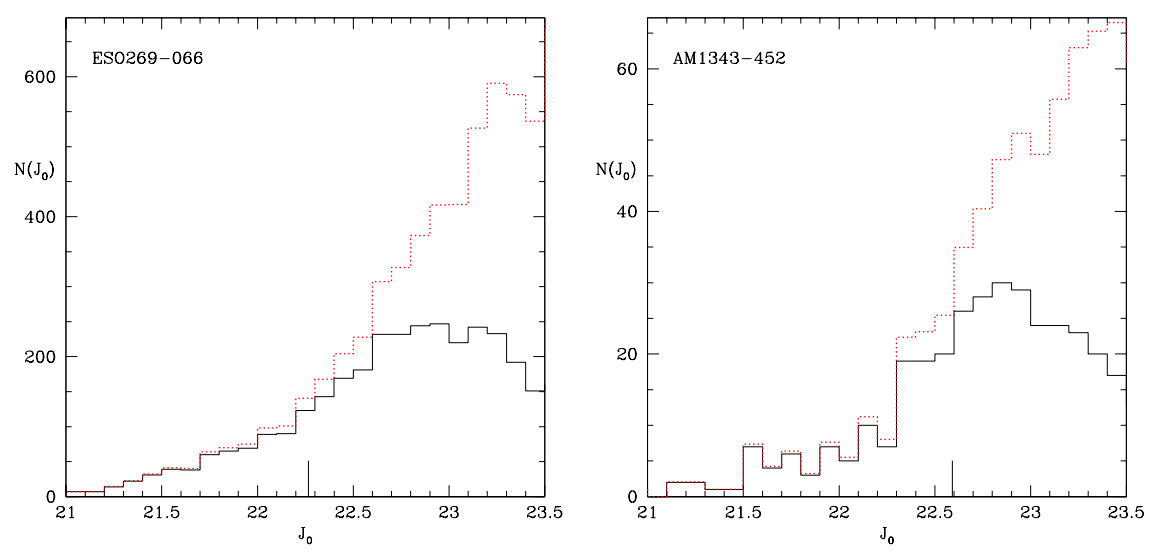

Figure 2. $J$ band LFs of ESO269-066 and AM1343-452. Solid histogram shows the observed $\mathrm{LF}$ with foreground contamination statistically subtracted. The dotted histogram is used for the completeness corrected LF. The short vertical line indicates the expected RGB tip break, assuming the distance modulus from Table 1 and metallicity determined from the RGB color at $M_{K}=-5.5$ (Valenti et al. 2004).

for AM1343-452. The larger uncertainty for the latter is due to larger distance modulus and consequently larger photometric errors and incompleteness.

The radial density profiles were used to determine the extent of each galaxy and the area where foreground stars dominated. These outer regions were used to statistically subtract the foreground stars. The subtraction is good for AM1343-452, while it works less well for ESO269-066, because of the larger radial extent of that galaxy. The $J$ band luminosity functions (LFs) of ESO269-066 and AM1343-452, with foreground contamination statistically subtracted, are presented in Fig. 2. The solid histogram is used for the observed, and the dotted line for the completeness corrected LF. The short vertical line indicates the expected magnitude of the RGB tip break, assuming the distance from the literature and the average metallicity for the RGB population.

The future work will include more detailed investigation of the intrinsic properties of the galaxies in our sample which will then be correlated with extrinsic properties like the local galaxy density in order to investigate the role of environment on their evolution. For more discussion of the environmental influences on the star formation histories of dwarf galaxies in groups the interested reader is referred to contributions of Da Costa and Karachentsev in this volume.

\section{Acknowledgements}

The participation of MR at the IAUC 198 was partially supported by an IAU Grant. The presented data were obtained at ESO Paranal Observatory within the observing programme 073.B-0131. HJ's and GDaC's work is supported in part by ARC Discovery Grant DP0343156.

\section{References}

Jerjen, H., Binggeli, B. \& Freeman, K.C., 2000, AJ 119, 593 (JBF00)

Jerjen, H., Freeman, K.C. \& Binggeli, B., 2000, $A J$ 119, 116 (JFB00)

Karachentsev, I.D., et al., 2002, A\&A 385, 21 (K+02)

Stetson, P.B., 1994, PASP 106, 250

Valenti, E., Ferraro, F.R. \& Origlia, L., 2004, MNRAS 351, 1204 